\title{
Physicomechanical Properties of the Extracellular Matrix of a Demineralized Bone
}

\author{
I. A. Kirilova ${ }^{1, a)}$, Yu. P. Sharkeev ${ }^{2,3, b)}$, S. V. Nikolaev ${ }^{4,5}$, V. T. Podorozhnaya ${ }^{1, c)}$, \\ P. V. Uvarkin ${ }^{2, d)}$ A. S. Ratushnyak ${ }^{5}$, and V. V. Chebodaeva ${ }^{3}$ \\ ${ }^{1}$ Novosibirsk Research Institute of Traumatology and Orthopedics n.a. Ya.L. Tsivyan, Novosibirsk, Russia \\ ${ }^{2}$ Institute of Strength Physics and Materials Science SB RAS, Tomsk, 634055 Russia \\ ${ }^{3}$ National Research Tomsk Polytechnic University, Tomsk, 634050 Russia \\ ${ }^{4}$ Institute of Cytology and Genetics, Novosibirsk, Russia \\ ${ }^{5}$ Design Technological Institute of Digital Techniques, Novosibirsk, Russia

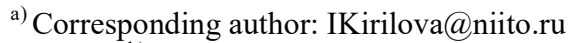 \\ b) sharkeev@ispms.tsc.ru \\ c)VPodorognaya@niito.ru \\ d) uvarkin@ispms.tsc.ru
}

\begin{abstract}
The article describes the results of a study of physicomechanical properties of a demineralized bone matrix of human cancellous and compact bones. A demineralized cancellous bone was shown to have the best characteristics of a porous system for colonization of matrices by cells. The ultimate stress and elasticity modulus of samples of demineralized femoral heads isolated in primary hip replacement was demonstrated to vary in wide ranges. The elasticity modulus ranged from 50 to $250 \mathrm{MPa}$, and the tensile strength varied from 1.1 to $5.5 \mathrm{MPa}$. Microhardness measurements by the recovered indentation method were not possible because of the viscoelastic properties of a bone material. To study the piezoelectric properties of samples, a measuring system was developed that comprised a measuring chamber with contact electrodes, a system for controlled sample loading, an amplifier-converter unit, and signal recording and processing software. The measurement results were used to determine the dependence of the signal amplitude on the dynamic deformation characteristics. The findings are discussed in terms of the relationship between the mechanical and electrical properties and the structure of the organic bone component.
\end{abstract}

\section{INTRODUCTION}

The problem of bone anatomic integrity and function restoration still remains topical [1-4]. The nature of regenerative processes is largely determined by the properties of materials used to fill bone defects. It is difficult to imagine modern medicine without biological implants that are used for reconstructive interventions in traumatology and orthopedics [3, 5-9], maxillofacial surgery [3, 10-13], and bone cancer [4, 7, 9].

The search for new materials and ways to affect osteogenesis to form an organotypic graft using osteoplastic materials (OPMs) is topical in modern medicine. OPMs fabricated from an allogeneic bone of various structure (cancellous, cortical, cortical-cancellous) start to be applied in various surgical areas. A promising technique of chemical pretreatment of an allogeneic bone appeared to be demineralization enabling fabrication of a demineralized bone lacking the mineral component $[2,9]$. At the same time, chemical pretreatment can alter the structure and properties of a native allogeneic bone and, therefore, its biological behavior upon implantation into the body.

To meet the needs of practical surgery, bioengineering constructs suitable for replacing extended bone defects are required. These constructs should have the following properties: preservation of the physical and anatomical features of an allogeneic bone, high strength, fast fixation in the implantation area, and the regenerative potential. These requirements can be met only by a stiff/elastic matrix containing recipient's bone cells capable of dividing, differentiating, and synthesizing the extracellular matrix that supports cell viability. 


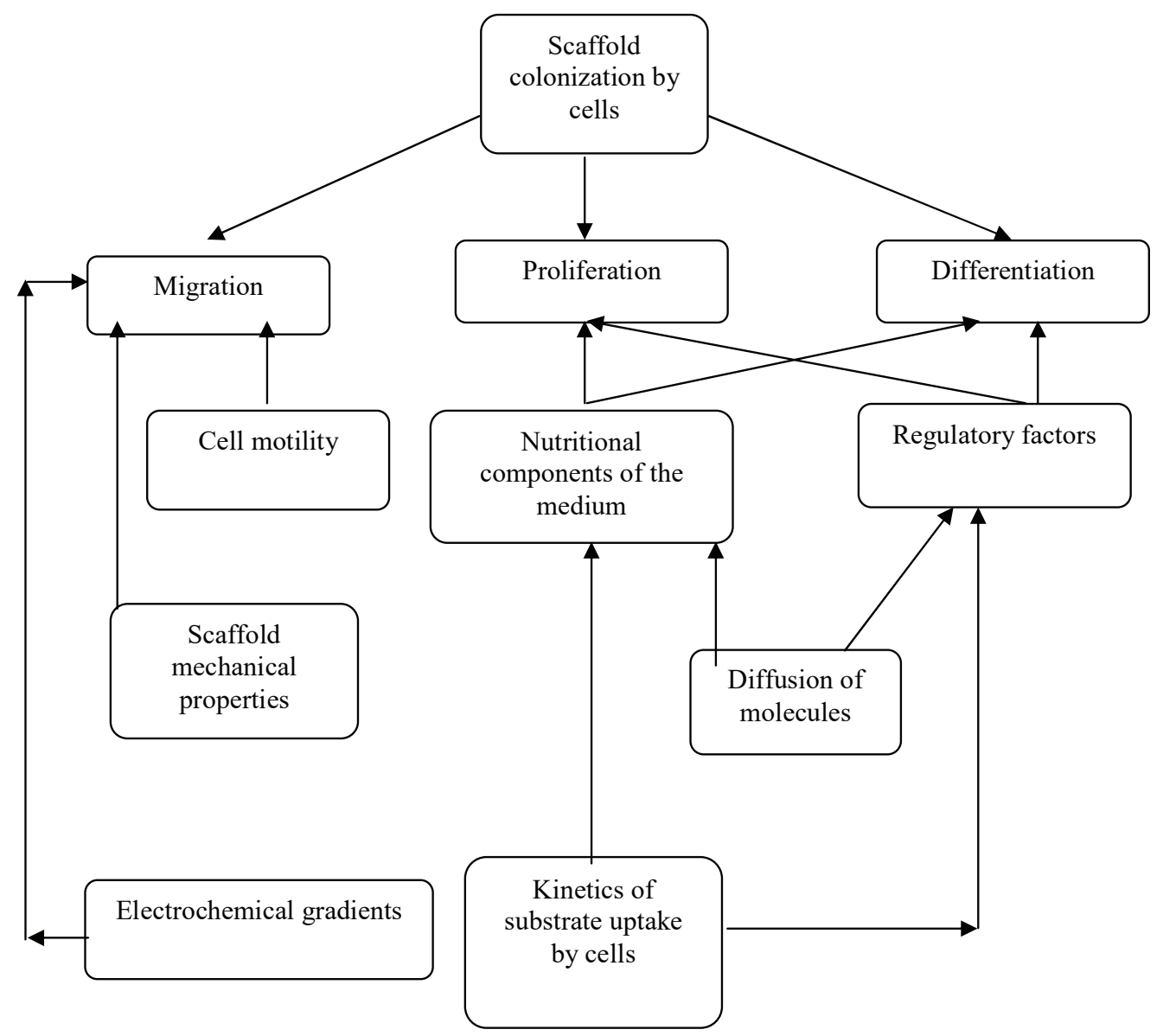

FIGURE 1. A diagram of scaffold colonization in implant bioengineering

The study aim was to investigate the effect of physicochemical properties of the demineralized bone matrix on osteogenic and chondrogenic cells to control their proliferation, differentiation, and taxis upon matrix colonization for regenerative medicine purposes.

The study aim was motivated by the authors' understanding of the process of scaffold colonization by cells in implant bioengineering (Fig. 1).

That is, colonization is the result of migration, proliferation, and differentiation of cells. Migration depends on cell motility and scaffold properties, mechanics, geometry, chemical composition of surfaces, etc. Differentiation and proliferation depend on the concentration of nutritional and regulatory components of the medium. The distribution of medium component concentrations is determined by diffusion of molecules and cell uptake of the components.

We suppose that these aims may be achieved if we learn to control cell functioning based on the knowledge of gene expression regulation (Fig. 2).

At the moment, we are interested in the following contours of gene expression regulation:

(i) Regulation by morphogens (regulatory molecules present in the medium).

(ii) Regulation by means of electric potentials and microcurrents.

(iii) Regulation by means of mechanical stresses and deformations.

The objectives generated on the basis of these considerations and prerequisites were as follows:

(i) To produce human demineralized bone scaffolds, conduct experiments on diffusion of substances into the scaffolds, and develop models of transport processes. 


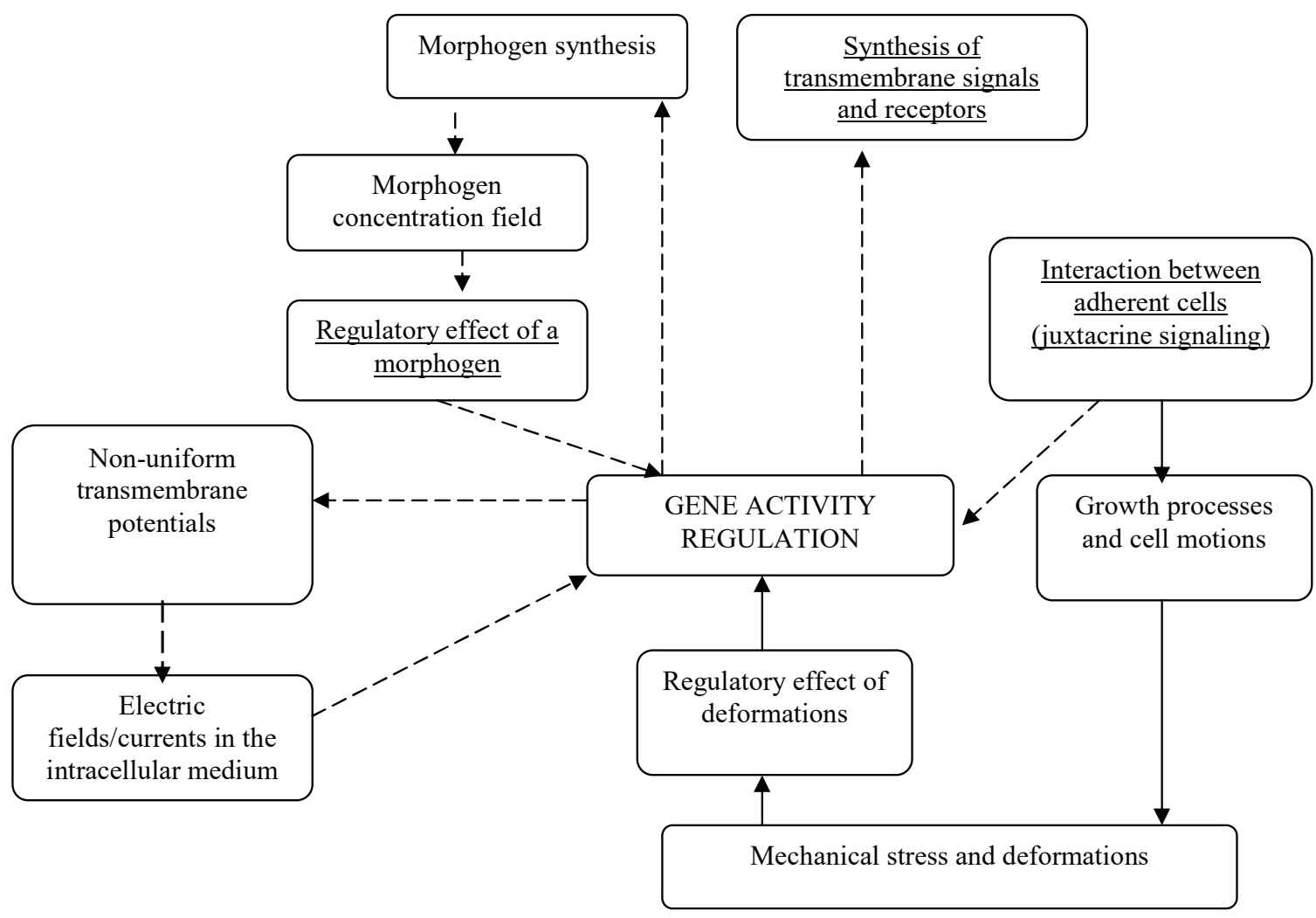

FIGURE 2. Contours of gene activity regulation

(ii) To study the mechanical properties of scaffolds. To use published data for selection of appropriate models of material mechanics.

(iii) To study the piezoelectric properties of scaffolds in order to use these properties for matrix stiffness modulation in experiments with cells.

\section{MATERIALS AND METHODS}

We investigated six samples of medical femoral head (FH) cuts resected during primary endoprosthetic replacement of the coxofemoral joint in coxarthrosis patients. Patients being bone tissue donors were $46-67$ years of age, with the mean age of 56 years. According to the approved regulations, blood of the bone tissue donors was tested for infectious agents (syphilis, HIV, and hepatitis B). If the test results were negative, tissue processing was started. At the first stage, the FH was cut along the radius with a step of $0.4-0.5 \mathrm{~cm}$. A sample with of $1.0 \times 3.0 \mathrm{~cm}$ in size was cut from the medial fragment. All donor bone tissues were washed in pure running water, delipidated with ether alcohol, and demineralization in a $0.6 \mathrm{~N} \mathrm{HCl}$ solution.

The microhardness was measured by a Duramin 5 microhardness tester with an indenter load of $245 \mathrm{mN}$. The microhardness was measured by the indent reconstruction method after indentation with a Vickers diamond tetrahedral pyramid having an angle of $136^{\circ}$ between the opposite facets. Three-point bending tests were performed using an Instron 3369 universal testing machine according to GOST 14019-80, with the loading rate of $0.1 \mathrm{~mm} / \mathrm{min}$ and the maximum load of $2 \mathrm{kN}$. A sample size in the three-point test was $10 \times 30 \times 4 \mathrm{~mm}$.

The bone tissue morphology was investigated using an Altami MET 1MT inverted metallographic microscope (Altami, Russia) in bright and dark fields and in polarized light. The bone tissue porosity was estimated from macroscopic images of study samples. The total porosity $\Pi$ was calculated according to the formula 


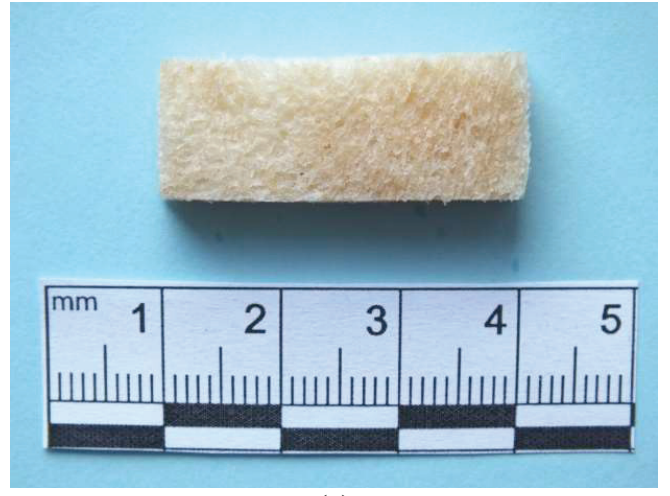

(a)

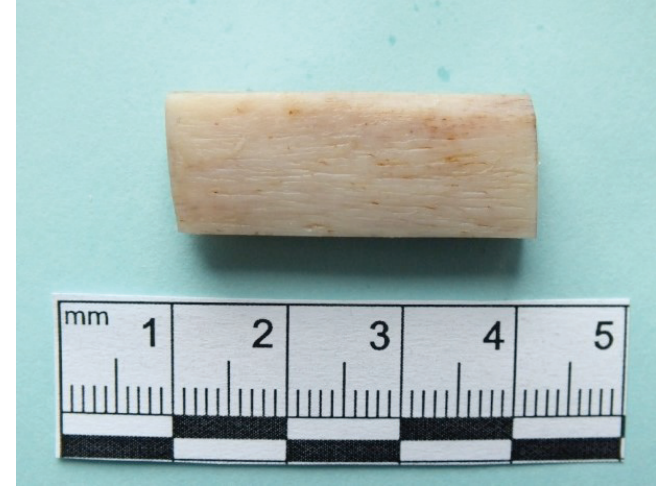

(b)

FIGURE 3. Macroimages of bone samples: (a) demineralized cancellous bone (DMCB), (b) demineralized cortical fragment (DMCF)

$$
\Pi=\frac{\sum l}{\sum L_{1}} 100 \%,
$$

where $L_{1}$ is the total length of secants, and $l$ is the length of secants overlapping pores measured in conventional units of a measuring device. To determine the elemental bone composition, a XRF-1800 sequential wavelength dispersive fluorescence X-ray spectrometer (Lab Center, Japan) was used.

\section{RESULTS AND DISCUSSION}

Demineralized cancellous and compact bone samples of the same size of $1.0 \times 3.0 \mathrm{~cm}$ have different macroscopic surface structures.

The morphology of all bone samples is represented by a porous structure. Pores occur over the entire bone. Figure 4 shows optical images of a bone fragment at different focal depths, which enables estimating internal pores of the sample (Fig. 5).

The morphology of compact bone samples is characterized by the widely spaced haversian canals, $20 \mu \mathrm{m}$ in size, with the intercanal distance ranging from 100 to $150 \mu \mathrm{m}$ and the porosity being close to 0 .

The demineralized cancellous bone structure has porosity of $50 \%$ or higher, with the mean pore diameter being most suitable for colonization of scaffolds by cells. Figure 6 shows a histogram of pore size distribution for a demineralized cancellous bone for the test samples. All distributions have a maximum whose position varies somewhat from sample to sample. Average porosity was $52 \%$ for samples 1 and 2, 55\% for 3, 4, and 5 and $67 \%$ for sample 6.
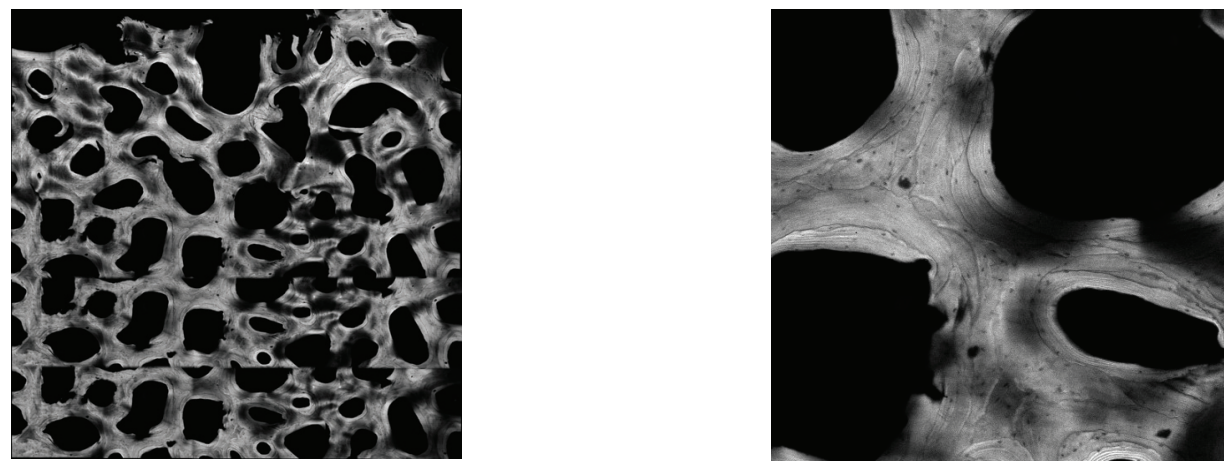

FIGURE 4. Optical images of deminiralized cancellous bone 

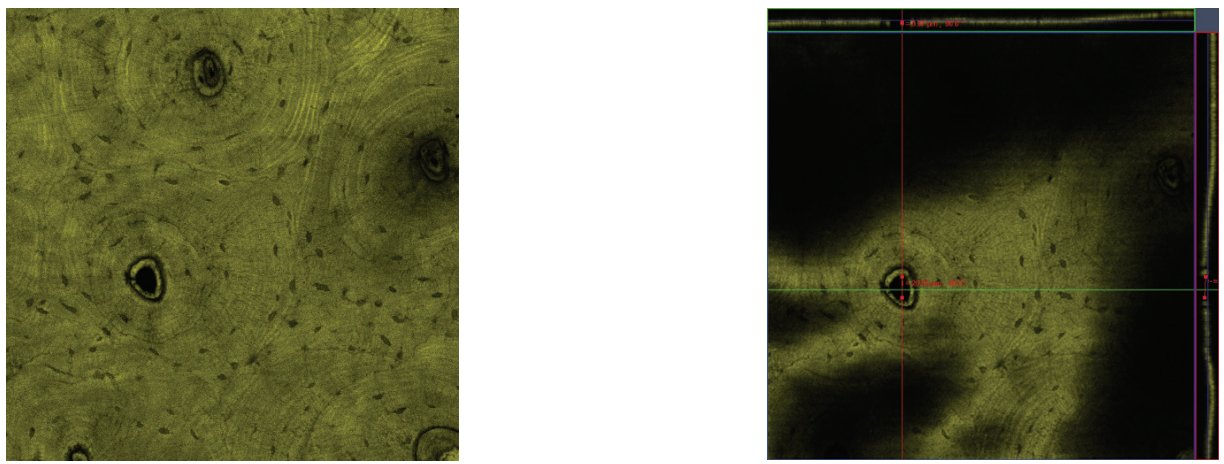

FIGURE 5. Optical images of a demineralized cortical bone

The literature $[5,11]$ indicate that samples with a pore size of $250 \mu \mathrm{m}$ or more are more preferable for using in colonization of scaffolds by osteoblasts, whose differentiated forms reach up to $30.0 \mu \mathrm{m}$ in size. An analysis of microimages revealed that the organic matrix of the cancellous bone has a pore size of about $0.5 \mathrm{~mm}$, with a low fraction of pores with a size of 10 to $100 \mu \mathrm{m}$.

Tables 1 and 2 show the mechanical properties of demineralized cancellous and cortical bone, respectively. Tensile strength for demineralized cancellous bone varied from 1.1 to $5.5 \mathrm{MPa}$ at the maximum deformation to $16.0 \%$. Bending Young's modulus varied from 49 to $248 \mathrm{MPa}$. In the case of demineralized cortical bone mechanical characteristics were lower. So strength limit varied between 1.1-5.5 MPa at a maximum deformation of $8.4 \%$. The Young's modulus in bending varied from 3 to $50 \mathrm{MPa}$. Lower characteristics occurred in cortical bone due to the presence of the porous skeleton structure in the absence of that of compact bone.

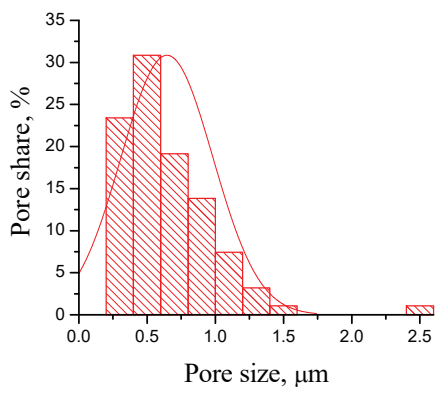

(a)

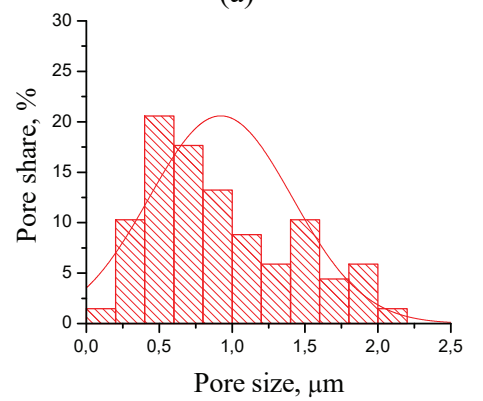

(d)

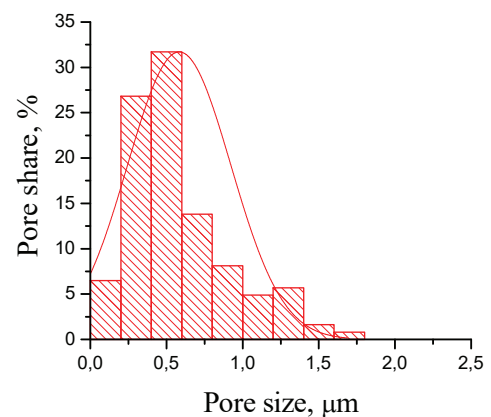

(b)

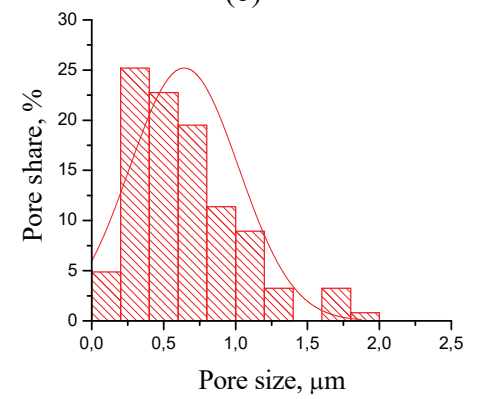

(e)

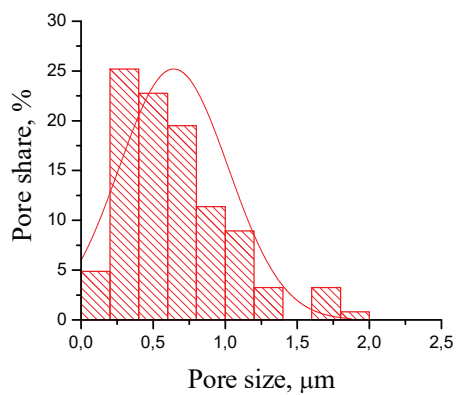

(c)

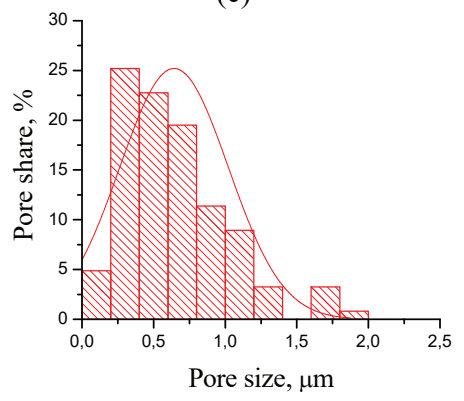

(f)

FIGURE 6. The pore distribution in a demineralized cancellous bone: (a) 1_1, (b) 1_2, (c) 1_3, (d) 1_4, (e) 1_5, (f) 1_6 
TABLE 1. Physicomechanical characteristics of a demineralized cancellous bone

\begin{tabular}{cccc}
\hline Sample & Ultimate stress $\boldsymbol{\sigma}_{\mathbf{B}}, \mathbf{M P a}$ & Deformation $\boldsymbol{\varepsilon}, \boldsymbol{\%}$ & Young's modulus, MPa \\
\hline 1 & 3.9 & 11.1 & 199 \\
\hline 2 & 2.0 & 13.1 & 75 \\
\hline 3 & 1.1 & 11.2 & 49 \\
\hline 4 & 5.5 & 16.0 & 220 \\
\hline 5 & 4.9 & 10.8 & 175 \\
\hline 6 & 4.4 & 11.5 & 248 \\
\hline
\end{tabular}

TABLE 2. Physicomechanical characteristics of a demineralized cortical bone

\begin{tabular}{cccc}
\hline Sample & Ultimate stress $\sigma_{\mathbf{B}}, \mathbf{M P a}$ & Deformation $\boldsymbol{\varepsilon}, \boldsymbol{\%}$ & Young's modulus, MPa \\
\hline 1 & 1.2 & 3.6 & 37 \\
\hline 2 & 0.4 & 7.5 & 12 \\
\hline 3 & 1.9 & 2.6 & 34 \\
\hline 4 & 0.4 & 4.1 & 14 \\
\hline 5 & 0.8 & 5.5 & 3 \\
\hline 6 & 0.8 & 8.4 & 11 \\
\hline 7 & 2.4 & 5.2 & 50 \\
\hline
\end{tabular}

Simulation of transport of morphogens and nutrients for controlling their spatial distribution requires knowledge of their diffusion coefficients for a particular sample. For this purpose, we mastered measurements of fluorescence recovery after photobleaching for optically transparent media and magnetic resonance imaging of the paramagnetic particle distribution, which can be used for optically opaque media. For a simple diffusion model, an inverse solution algorithm was developed to estimate the diffusion coefficient by analyzing a series of images of the diffusing substance distribution in a sample. Diffusion coefficients of several matrices were predetermined in pilot experiments. We developed an experimental setup for measurement of the piezoelectric properties of samples (Fig. 7) and recorded piezoelectric potentials of a demineralized autogenous compact bone sample. The shape and amplitude of piezoelectric potentials depend on the sample microstructure and humidity. An indirect estimation of humidity was performed by measurement of electrical conductivity of the sample area located under the electrodes.

A flowchart of the hardware-software measurement complex is as follows: a measuring chamber with a sample (1), a preamplifier-coupler (2), a manipulator (3), a measuring-controlling complex (4).

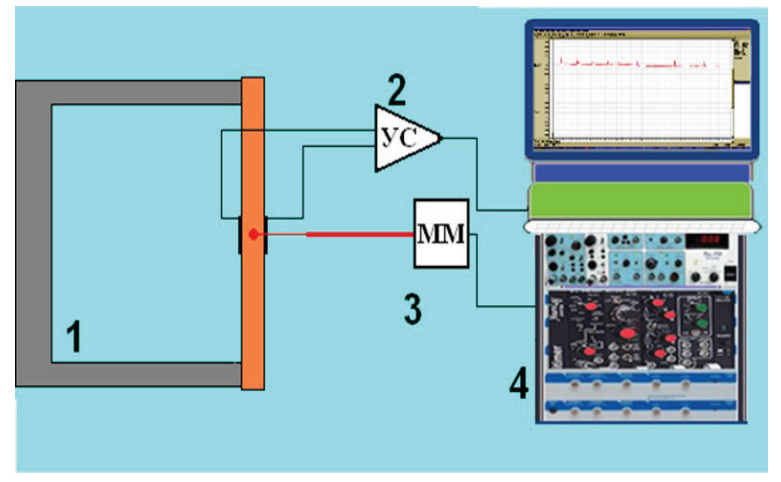

FIGURE 7. An experimental setup for measurement of bone piezoelectric properties 


\section{CONCLUSION}

The mechanical and electrical properties are interrelated and depend on the bone tissue type (compact, cancellous). Tensile strength and modulus of demineralized bone tissue samples vary widely. In the case of demineralized head of the femur mechanical properties slightly lower performance of demineralized cortical bone, which is associated with the presence of the frame structure at the latest. The porosity of demineralized cancellous bone significantly exceeds the porosity of the compact bone and is $67 \%$. Demineralized spongy bone has the best porosity characteristics for settlement matrices cells. Bone has piezoelectric properties, and the shape and amplitude of the piezoelectric potentials depend on the microstructure of bone.

\section{ACKNOWLEDGMENTS}

This work was supported by the Russian Foundation for Basic Research project No. 15-29-04875.

The study reported in this article was conducted according to accepted ethical guidelines involving research in humans and/or animals and was approved by an appropriate institution or national research organization. The study is compliant with the ethical standards as currently outlined in the Declaration of Helsinki. All individual participants discussed in this study, or for whom any identifying information or image has been presented, have freely given their informed written consent for such information and/or image to be included in the published article.

\section{REFERENCES}

1. Z. Liao, C. H. Wang, and W. L. Cui, J. Invest. Surg. 11, 1-10 (2016).

2. I. A. Kirilova, N. G. Fomichev, and V. T. Podorozhnaya, Spine Surgery: Digest 1 (2013), http://www. spinesurgery.ru/Digest.

3. R. T. Nigmatullin, D. A. Shcherbakov, L. M. Musina, and A. A. Tkachev, Medical Bulletin Bashkortostan 7(4), 78-83 (2012).

4. S. V. Dianov and A. N. Tarasov, Traumatology Orthopedics Rus. 3, 130-132 (2009).

5. S. Hofer, S. S. Leopold, and J. Jacobs, ASTM Int., 68-95 (2003).

6. M. R. Major, V. W. Wong, E. R. Nelson, M. T. Longaker, and G. C. Gurtner, Plast. Reconstr. Surg. 135(5), 1489-1498 (2015), doi 10.1097/PRS.0000000000001193.

7. V. T. Podorozhnaya, I. A. Kirilova, Yu. P. Sharkeev, and E. V. Legostaeva, Izv. Vuzov. Phys. 12/3, 14-20 (2013).

8. I. A. Kirilova, et al., AIP Conference Proceedings 1688, 030005 (2015), doi 10.1063/1.4936000.

9. http://dx.doi.org/10.1063/1.4936000, AIP Publishing.

10. F. P. Cammisa, G. Lowery, S. R. Garfin, F. H. Geisler, P. M. Klara, R. A. McGuire, W. R. Sassard, H. Stubbs, and J. E. Block, Spine 29, 660-666 (2004).

11. A. I. Shvets and V. K. Ivchenko, Orthopedics, Traumatology Prosthetics 3, 66-69 (2008).

12. I. Parisay, J. Ghoddusi, and M. Forghani, Iran Endod J. 10(1), 6-15 (2015).

13. A. I. Grudianov and A. V. Nikolaev, Dentistry 95(1), 40-43 (2016). 